\title{
Atmospheric production of radiation belt light isotopes
}

\author{
R. S. Selesnick ${ }^{1}$ and R. A. Mewaldt \\ California Institute of Technology, Pasadena
}

\begin{abstract}
Nuclear interactions between inner zone protons and atoms in the upper atmosphere produce energetic $\mathrm{H}$ and $\mathrm{He}$ nuclei that are an additional radiation belt source. We calculate production rates of these isotopes from models of the inner zone proton intensity, the upper atmosphere drift-averaged composition and densities, and cross sections for the various interaction processes. For comparison with observations of radiation belt $\mathrm{H}$ and $\mathrm{He}$ isotopes, the production rates are combined with a model of the energy loss rate in the residual atmosphere to calculate particle intensities. Although the calculations are in principle straightforward, they depend on a detailed knowledge of the various model inputs, including models for radiation belt protons, and may also depend on the phase of the solar cycle. On the other hand, the results of the calculations, when compared with the observational data, can provide useful tests of the model inputs. Initial results show that the atmosphere is a significant source for inner zone ${ }^{4} \mathrm{He},{ }^{3} \mathrm{He}$, and ${ }^{2} \mathrm{H}$.
\end{abstract}

\section{Introduction}

The inner radiation belt is composed primarily of protons that were produced locally by the cosmic ray albedo neutron decay (CRAND) process, as a result of collisions between cosmic rays and the neutral atmosphere. The trapped protons similarly create a secondary source of trapped particles by their own nuclear interactions with the atmosphere. This process is less significant than CRAND for producing protons because the particles must be injected directly, whereas the neutrons can propagate to high altitudes, where the atmospheric density is low, before decaying. However, because CRAND produces only protons and electrons, the secondary process can be a significant source of other trapped particles such as isotopes of $\mathrm{H}$ and $\mathrm{He}$.

The CRAND source has been the subject of several theoretical calculations for comparison with the trapped proton data [e.g., Dragt, 1971; Farley and Walt, 1971; Jentsch and Wibberenz, 1980; Jentsch, 1981]. The secondary source has not been studied in detail, probably because of a lack of data on the composition of highenergy trapped ions. However, the new data from SAMPEX [Cummings et al., 1995; Looper et al., 1995] is sufficient for evaluating some results from models of the secondary source. Although the calculation is analogous to the CRAND case, it is complicated by the need to calculate the source function due to each of the many possible nuclear interactions. In the CRAND calcu-

\footnotetext{
${ }^{1}$ Now at The Aerospace Corporation, Los Angeles.
}

Copyright 1996 by the American Geophysical Union.

Paper number 96JA01746.

0148-0227/96/96JA-01746\$09.00 lations the neutron source was generally an empirical model based on neutron flux measurements.

\section{Calculations}

If the atmospheric production of trapped particles is balanced only by ionization energy loss in the atmosphere, then their intensity $j$ satisfies a continuity equation [Jentsch and Wibberenz, 1980]

$$
\frac{1}{v} \frac{\partial j}{\partial t}=S+\frac{\partial}{\partial E}\left(j\left|\frac{d E}{d x}\right|\right)
$$

where $v=d x / d t$ is the (nonrelativistic) speed of the trapped particle at time $t$ and kinetic energy $E$. The production rate in $\left(\mathrm{cm}^{3} \mathrm{~s} \mathrm{sr} \mathrm{MeV}\right)^{-1}$ is $S$. The intensity $j$ can be time dependent through $S$ which generally varies due to the solar cycle. In the steady state case or if the particle lifetimes are short compared to the 11-year solar cycle, the solution is

$$
j=\frac{1}{\left|\frac{d E}{d x}\right|} \int_{E}^{\infty} S d E
$$

The integral should be cutoff at the maximum energy of adiabatically trapped particles, but this is not significant for a sufficiently soft spectrum. The solution (2) also applies to particles with lifetimes that are long compared to the solar cycle time if $S$ is interpreted as the solar cycle average value. For cases where the lifetimes are comparable to the solar cycle time, a timedependent solution is possible [Jentsch and Wibberenz, 1980]. For protons, the continuity equation (1) is valid for low $L$ shells $(L \lesssim 1.3$ ) beyond which radial diffusion from an external source becomes significant at low energies $(\lesssim 30 \mathrm{MeV})$ [Jentsch, 1981]. 


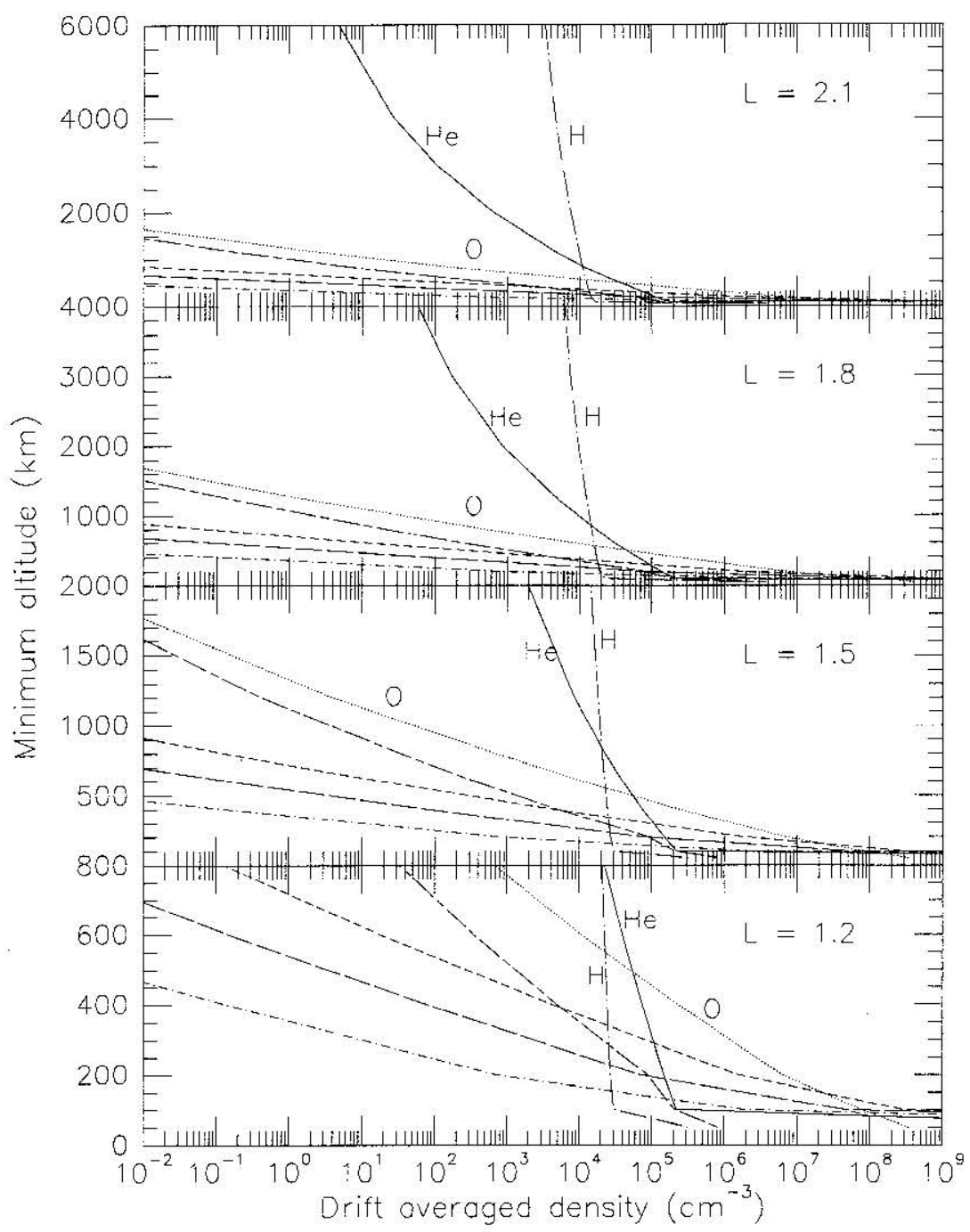

Figure 1. Drift-averaged atmospheric densities versus minimum altitude of the particle trajectory from the MSIS-86 and IGRF-92 models at selected $L$ shells and for solar average $(F 10.7=140)$ conditions $(\mathrm{O}$, dotted; $\mathrm{O}_{2}$, long dashed; $\mathrm{H}$, dotted-long dashed; $\mathrm{He}$, solid; $\mathrm{N}_{2}$, short dashed; $\mathrm{Ar}$, dotted-dashed; $\mathrm{N}$, dashedlong dashed).

The production rate of secondaries is

$$
S=\sum_{i} \int d E_{p} \int d \Omega_{p} n_{i} j_{p} \frac{d^{2} \sigma_{i}}{d \Omega d E}
$$

The summation extends over all interactions that lead to a given type of secondary particle and the integrals cover the range of proton energies $E_{p}$ and solid angle $\Omega_{p}$ that kinematically can produce secondaries with energy $E$, pitch angle $\alpha$ in a solid angle $\Omega$, and at a given $L$ shell. The atmospheric density $n_{i}$ of target atoms for the interaction $i$ is averaged over the drift path of protons for each $L$ and proton pitch angle $\alpha_{p}$. The proton intensity is $j_{p}$, and the cross section for interaction $i$ is $\sigma_{i}$. Both $n_{i}$ and $j_{p}$ may be functions of time due to solar cycle variations. Calculating $S$ from (3) requires knowledge of the atmospheric densities, the trapped proton intensities and drift paths, and the interaction cross sections. In addition, calculating $j$ from (2) requires knowledge of the energy loss rate in the atmosphere.

For the atmosphere we use the mass spectrometer incoherent scatter 1986 (MSIS-86) neutral atmosphere model [Hedin, 1987] as encoded by the National Space Science Data Center (NSSDC), which provides number densities of $\mathrm{He}, \mathrm{O}, \mathrm{N}_{2}, \mathrm{O}_{2}, \mathrm{Ar}, \mathrm{H}$, and $\mathrm{N}$ as functions of day of year, local time, altitude, geodetic latitude and longitude, solar 10.7-cm flux ( $F 10.7)$ for the previous day and a 3 -month average, and the magnetic $A_{p}$ index. For the Earth's magnetic field we use the International Geomagnetic Reference Field (IGRF) model [Langel, 1991] from the NSSDC extrapolated to 1992. To calculate the drift averaged densities for each element, trajectories of $100-\mathrm{MeV}$ protons were calculated numerically for a given $L$ shell starting from the minimum magnetic field $B$ on that $L$ shell at a given altitude and continuing for one complete drift in longitude around the Earth. The minimum $B$ was converted to an equatorial pitch 
angle and the starting altitude was varied to provide the drift-averaged densities as a function of $\alpha_{p}$. A typical value of $F 10.7=140$ was used to simulate solar average conditions. Results for selected $L$ shells are shown in Figure 1.

The drift-averaged atmospheric densities were also used to calculate $d E / d x$, the energy loss rate in the atmosphere, using the formulas compiled by Salamon [1980] and weighting the energy loss rates in each atmospheric constituent according to the drift-averaged densities at each altitude. We assume that the secondary particles have no bound electrons. Energy loss to free electrons in the ionosphere and plasmasphere is not included. Trapped ${ }^{4} \mathrm{He}$ lifetimes calculated from $d E / d x$ are shown in Figure 2. They are generally short relative to the solar cycle at low altitudes (small equatorial pitch angles) but can be long at high altitudes and $L$ shells.

The proton intensities were derived from the empirical NASA AP-8 models obtained from NSSDC. These provide proton omnidirectional integral intensities, $J_{p}$, as a function of $L$ and $B / B_{0}$, the ratio of local to equatorial magnetic fields, for solar minimum and solar maximum conditions, although significant solar cycle variations were not found for our region of interest. The equatorial directional differential intensity was calculated by numerically evaluating

$$
j_{p}=\frac{1}{2 \pi^{2}} \frac{\partial^{2}}{\partial E \partial x} \int_{0}^{x} \frac{J_{p}\left(E, x^{\prime}\right)}{\left(x-x^{\prime}\right)^{1 / 2}} d x^{\prime}
$$

where $x=B_{0} / B$. Sample proton energy spectra are shown in Figure 3.

The final inputs to the source function (3) are the cross-section data for a given nuclear interaction. This should be differential in energy and solid angle. If the interaction has only two ingoing and two outgoing particles, then the kinematics can be used to simplify the calculation. For the reaction designated $1(2,3) 4$ involving particles of rest mass $m_{1}, m_{2}, m_{3}$, and $m_{4}$, where $m_{1}$ collides with $m_{2}$ which is at rest in the laboratory system, the initial 4-momenta are $\left(E_{1}, \mathbf{p}_{1}\right),\left(m_{2}, 0\right)$ in the lab and $\left(\epsilon_{1}, \mathbf{k}\right),\left(\epsilon_{2},-\mathbf{k}\right)$ in the center-of-mass, while the final four-momenta are $\left(E_{3}, \mathbf{p}_{3}\right),\left(E_{4}, \mathbf{p}_{4}\right)$ and $\left(\epsilon_{3}, \mathbf{k}^{\prime}\right),\left(\epsilon_{4},-\mathbf{k}^{\prime}\right)$ respectively. If $m_{4}$ is the secondary particle of interest, then the scattering angles in the center of mass, $\Theta$, and the lab, $\theta_{4}$ are related to the energies by the Lorentz transformation

$$
E_{4}=\gamma \epsilon_{4}-\gamma \beta k^{\prime} \cos \Theta
$$

and to each other by

$$
\tan \theta_{4}=\frac{\sin \Theta}{\gamma\left(-\cos \Theta+\frac{\beta}{\beta_{4}}\right)}
$$

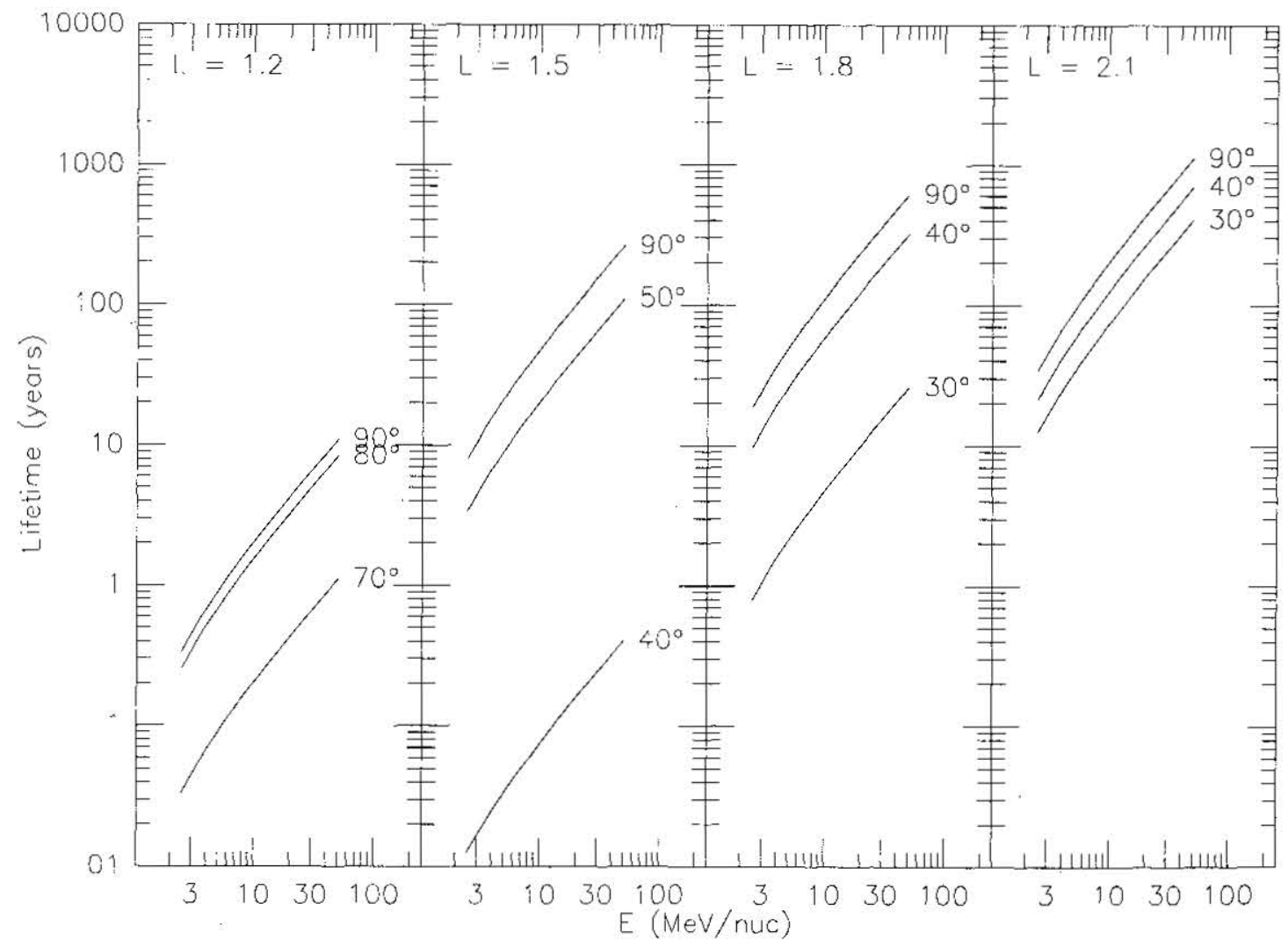

Figure 2. Lifetimes against energy loss in the atmosphere for trapped ${ }^{4}$ He at selected $L$ shells and labeled equatorial pitch angles. 


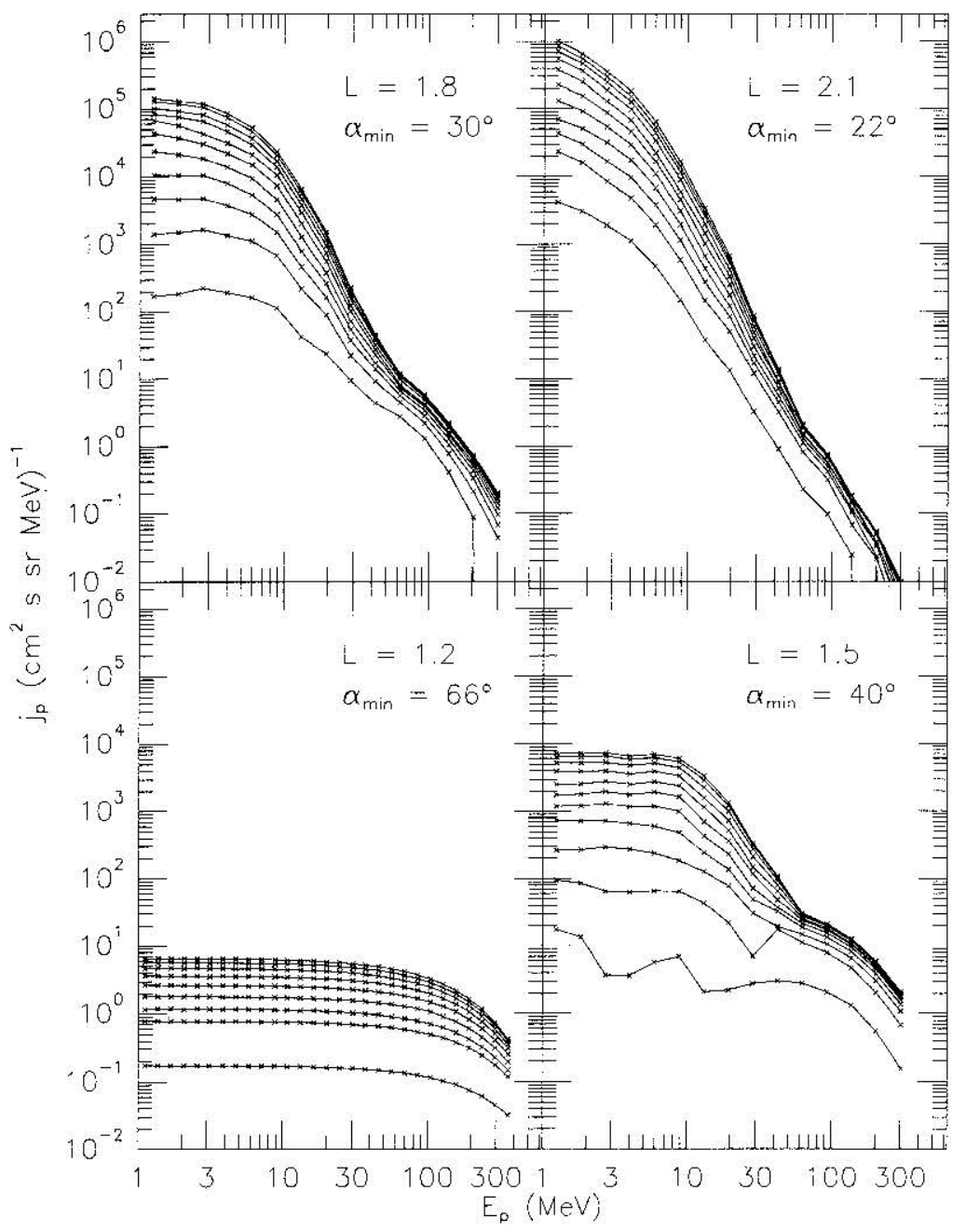

Figure 3. Proton energy spectra for selected $L$ shells from the AP-8 model. The curves for each $L$ are at equally spaced pitch angles varying from the labeled minimum $\alpha$ to $90^{\circ}$. Some smoothing was done for the $L=1.2$ curves.

where $\beta=p_{1} /(\gamma E)$ and $\gamma=\left(E_{1}+m_{2}\right) / E$ are the center of mass speed and Lorentz factor, $E=\left(2 E_{1} m_{2}+m_{1}^{2}+\right.$ $\left.m_{2}^{2}\right)^{1 / 2}$ and $\beta_{4}=k^{\prime} / \epsilon_{4}$ are the total energy and $m_{4}$ speed in the center-of-mass. From (5)

$$
\frac{d \sigma}{d E_{4}}=-\frac{2 \pi}{\gamma \beta k^{\prime}} \frac{d \sigma}{d \Omega_{c}}
$$

where $\Omega_{c}$ is the center-of-mass solid angle. In this case the double differential cross section can be expressed in terms of either of the single differential cross sections. For example,

$$
\frac{d^{2} \sigma}{d \Omega d E_{4}}=\frac{d \sigma}{d E_{4}} \frac{\delta\left(\theta_{4}-\theta_{4}(E)\right)}{2 \pi \sin \theta_{4}}
$$

where $\theta_{4}(E)$ is given by $(6)$.

To do the source integral (3) over the $\delta$ function in (8), we change variables from $\alpha_{p}$ and the proton gyrophase angle to $\theta$ and the initial proton phase angle $\phi_{p}$ around the secondary particle direction by a coordinate system rotation:

$$
S=\sum_{i} \int d E_{p} \frac{d \sigma_{i}}{d E} \frac{1}{\pi} \int_{0}^{\pi} d \phi_{p} n_{i} j_{p}
$$

where $\cos \phi_{p}=\left(\cos \alpha_{p}-\cos \alpha \cos \theta\right) /(\sin \alpha \sin \theta)$ and $\theta$ is the scattering angle $\theta_{4}$ from (6). Note that if $n_{i}$ and $j_{p}$ are independent of $\alpha_{p}$ then (9) leads to the expected result

$$
S=\sum_{i} \int d E_{p} n_{i} j_{p} \frac{d \sigma_{i}}{d E}
$$

for the source function due to an isotropic proton flux in a homogeneous atmosphere.

The first interaction that we consider is $\mathrm{p}\left({ }^{4} \mathrm{He}, \mathrm{p}\right)^{4} \mathrm{He}$, elastic scattering of protons on atmospheric ${ }^{4} \mathrm{He}$. The maximum kinetic energy of the recoil ${ }^{4} \mathrm{He}$ is approximately $16 / 25$ times the proton kinetic energy, so that a $100 \mathrm{MeV}$ proton can produce a ${ }^{4} \mathrm{He}$ of up to 16 $\mathrm{MeV} /$ nucleon. Cross-section data are shown in Figure 4 from various sources listed in the figure caption. They are generally peaked in the direction where protons are forward scattered, but the most efficient direction 


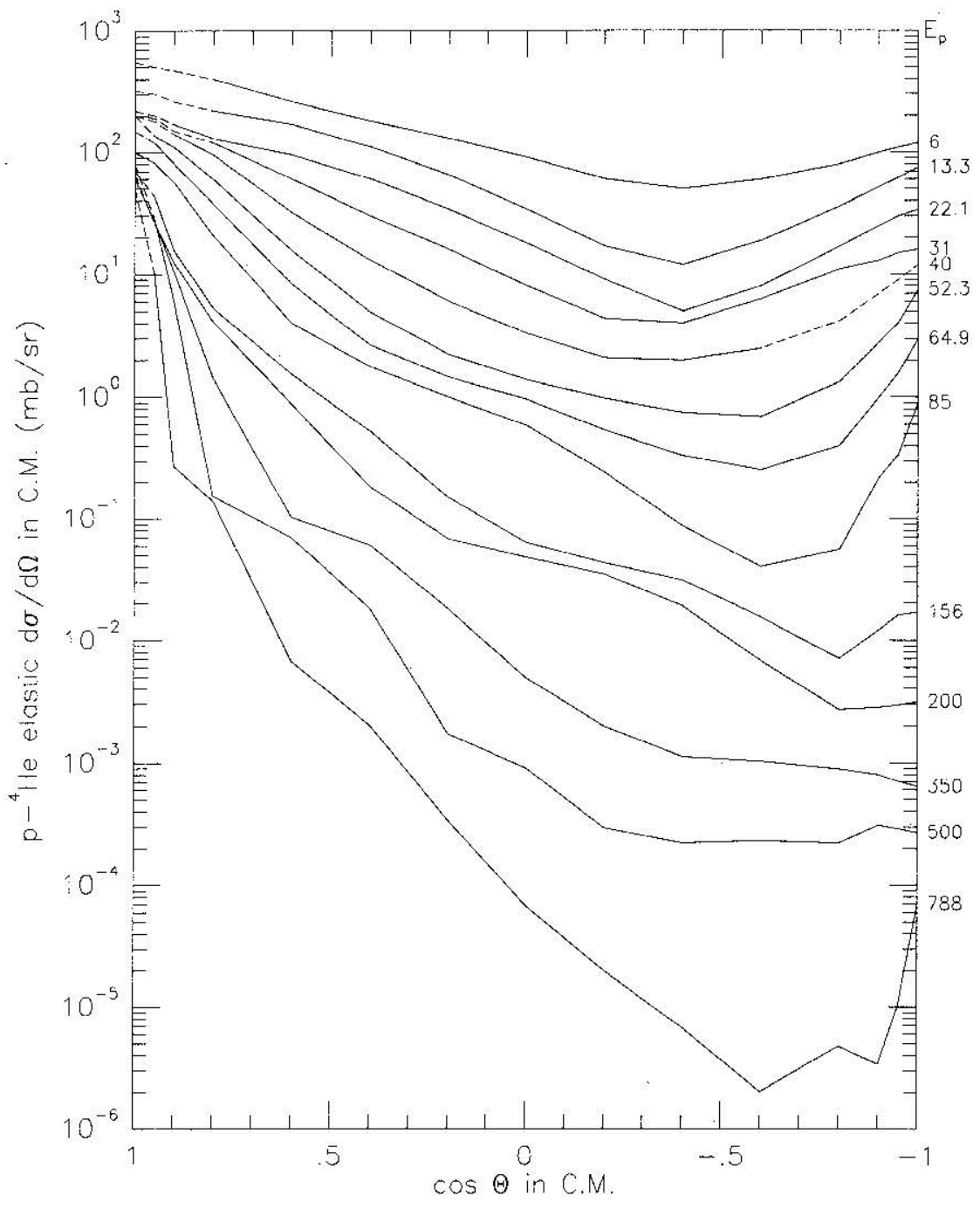

Figure 4. Cross sections versus scattering angle in the center of mass for $\mathrm{p}^{4} \mathrm{He}$ elastic scattering [Meyer et al., 1972, Votta et al., 1974; Comparat et al., 1975; Fong et al., 1978; McCamis et al., 1978; Imai et al., 1979; Moss

for trapping the recoil ${ }^{4} \mathrm{He}$ is where the protons are scattered backward $(\cos \Theta=-1)$ and the ${ }^{4} \mathrm{He}$ follow the original proton trajectories. However, small and intermediate angle scattering can also lead to significant particle trapping if the pitch angle is approximately conserved, especially at the high proton energies where the cross sections are strongly forward-peaked. Therefore it is necessary to include the full angular dependencies in the source integral (9).

Results of the calculation using (9) and (2) for elastic scattering of ${ }^{4} \mathrm{He}$ are shown in Figure 5. The ${ }^{4} \mathrm{He}$ intensity at a given energy per nucleon varies with equatorial pitch angle due to the corresponding variation in the relative concentration of ${ }^{4} \mathrm{He}$ in the driftaveraged atmosphere. It varies with $L$ primarily due to the variation in the proton interisity. The pitch angle distribution changes from being strongly peaked at $90^{\circ}$ for $L=1.2$ to being peaked near the edge of the loss cone for $L=2.1$. et al., 1980]. Laboratory proton energies are labeled to the right of each curve in $\mathrm{MeV}$. Dashed curve segments are extrapolations.

Measurements of the ${ }^{4} \mathrm{He}$ energy spectra from the MAST instrument on SAMPEX are also shown in Figure 5 [Cummings et al., 1995]. These are averaged over the local pitch angles, but because they were taken at the SAMPEX altitude of 520 to $670 \mathrm{~km}$, only particles with equatorial pitch angles that mirror at or below this altitude are included. Note that the SAMPEX orbit is in the altitude range where $\mathrm{He}$ is the dominant driftaveraged atmospheric component (Figure 1). Therefore the model intensities from an atmospheric He source are highest in this altitude range, and the equatorial pitch angles that are most comparable to those observed by SAMPEX generally correspond to the model curves with the highest intensities.

We next consider the pickup reaction $\mathrm{p}\left({ }^{4} \mathrm{He}, \mathrm{d}\right)^{3} \mathrm{He}$. Cross-section data are shown in Figure 6 with the convention that $\Theta$ is the angle between the $p$ and $d$ trajectories. The backward direction $(\cos \Theta=-1)$ is now most efficient for trapping ${ }^{3} \mathrm{He}$ while the forward 


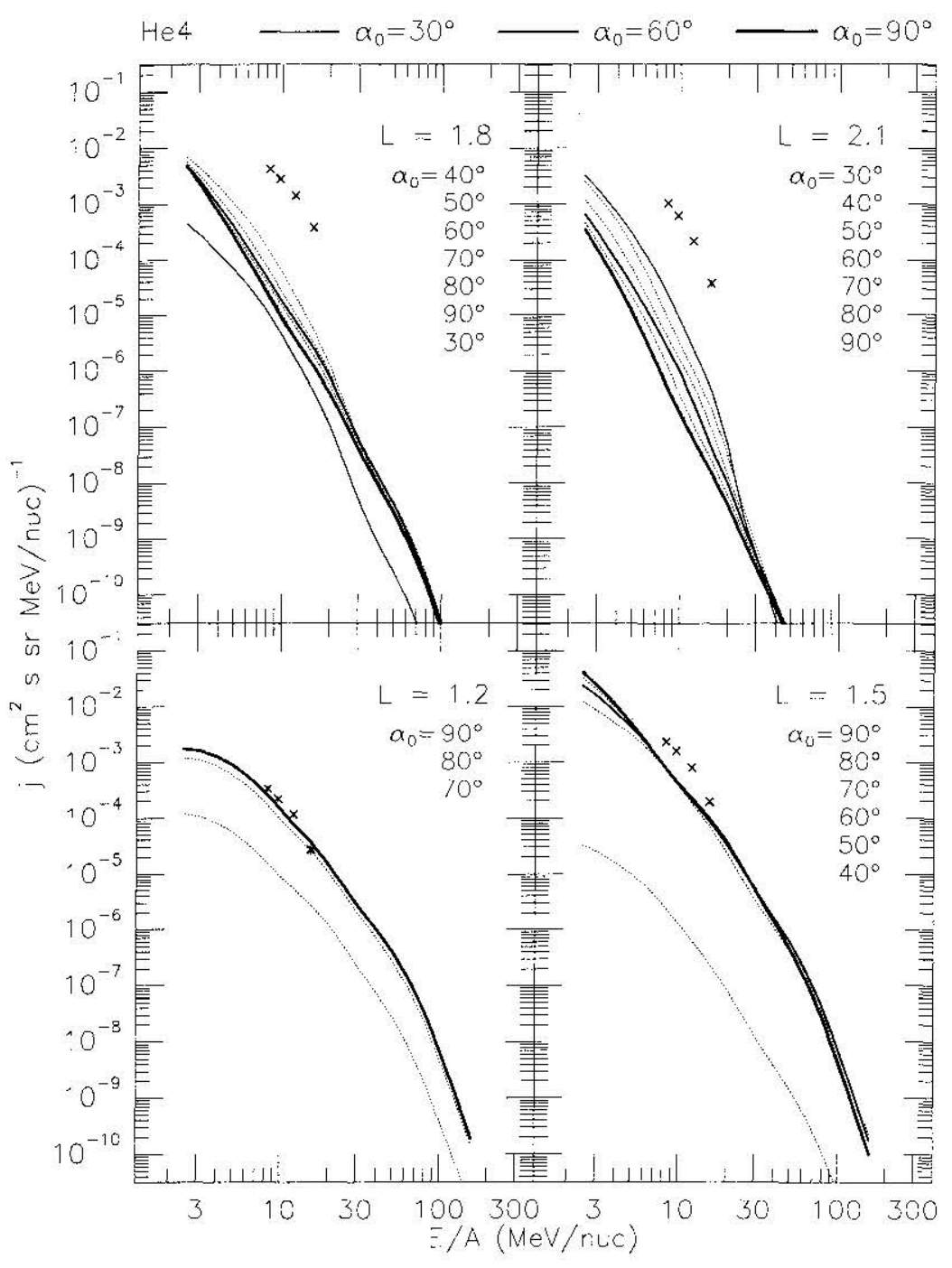

Figure 5. Intensity of ${ }^{4} \mathrm{He}$ from elastic scattering of protons as a function of energy-per-nucleon at average solar conditions for the labeled $L$ shells. The equatorial pitch angles $\alpha_{0}$ vary from $30^{\circ}$ (off scale for low $L$ ) to $90^{\circ}$ in steps of $10^{\circ}$. The solid curves correspond to the $\alpha_{0}$ values labeled at the top. The $\alpha_{0}$ values listed in

direction ( $\cos \Theta=1$ ) is most efficient for trapping $d$. The ${ }^{3} \mathrm{He}$ and $\mathrm{d}$ intensities are shown in Figures 7 and 8 respectively. The $d$ intensities are higher than the ${ }^{3} \mathrm{He}$ intensities because the cross sections are forward peaked. Data points in these Figures are again from the MAST instrument on SAMPEX for ${ }^{3} \mathrm{He}$ [Cummings et al., 1995] and from the PET instrument on SAMPEX for $\mathrm{d}$ [Looper et al., 1995]. The ratio of the ${ }^{3} \mathrm{He}$ and ${ }^{4} \mathrm{He}$ intensities is shown in Figure 9 and the ratio of the $\mathrm{d}$ and $\mathrm{p}$ intensities is shown in Figure 10.

There are other reactions that can also produce the isotopes considered above. For example, protons can collide with atmospheric $\mathrm{O}$, which is relatively dense at low altitudes, producing evaporation and direct knockout products including ${ }^{4} \mathrm{He},{ }^{3} \mathrm{He}$, and $\mathrm{d}$. The kinematics are not determined as in (5). Instead, cross sections and the energy distribution of the products are measured. each panel are in order of decreasing intensity from top to bottom at $10 \mathrm{MeV} /$ nucleon. The data points are from SAMPEX/MAST ${ }^{4} \mathrm{He}$ observations averaged over local pitch angles at $\sim 600 \mathrm{~km}$ altitude [Cummings et al., 1995].

However, the data are sparse and several approximations must be made. Detailed measurements at $E_{p}=90$ $\mathrm{MeV}$ have been made by Wu et al. [1979]. They found that the evaporation products are generally isotropic and low energy, so they are not significant here, while the knock-out products are forward-peaked and higher energy. To simplify the calculation, we assume that they are produced in the forward direction only

$$
\frac{d^{2} \sigma}{d \Omega d E}=\sigma\left(E_{p}\right) \frac{\delta(\theta)}{2 \pi \sin \theta} F\left(E_{p}, E\right)
$$

For the energy distribution $F$ we assume an exponential independent of $E_{p}$ except for a cutoff at the maximum energy $E_{\max }$ which differs from $E_{p}$ by the binding energy of the knock-out product in the original nucleus

$$
F\left(E_{p}, E\right)=\frac{1}{E_{0}} \frac{e^{-E / E_{0}}}{1-e^{-E_{\max } / E_{0}}} H\left(E_{\max }-E\right)
$$




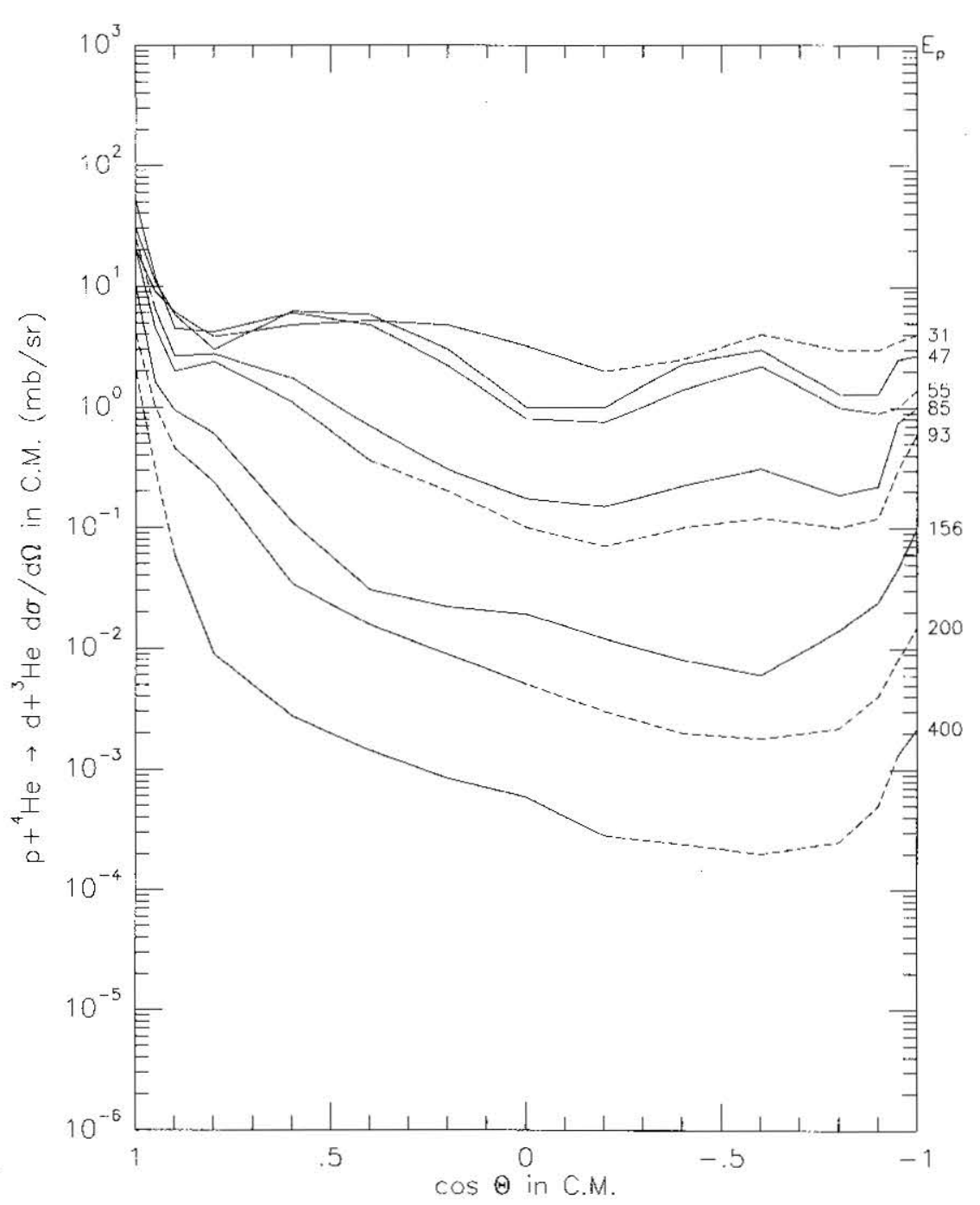

Figure 6. Cross sections versus scattering angle in the center of mass for the reaction $\mathrm{p}\left({ }^{4} \mathrm{He}, \mathrm{d}\right)^{3} \mathrm{He}[$ Meyer et al., 1972; Votta et al., 1974; Alons et al., 1986]. Laboratory proton energies are labeled to the right of each curve in $\mathrm{MeV}$. Dashed curve segments are extrapolations.

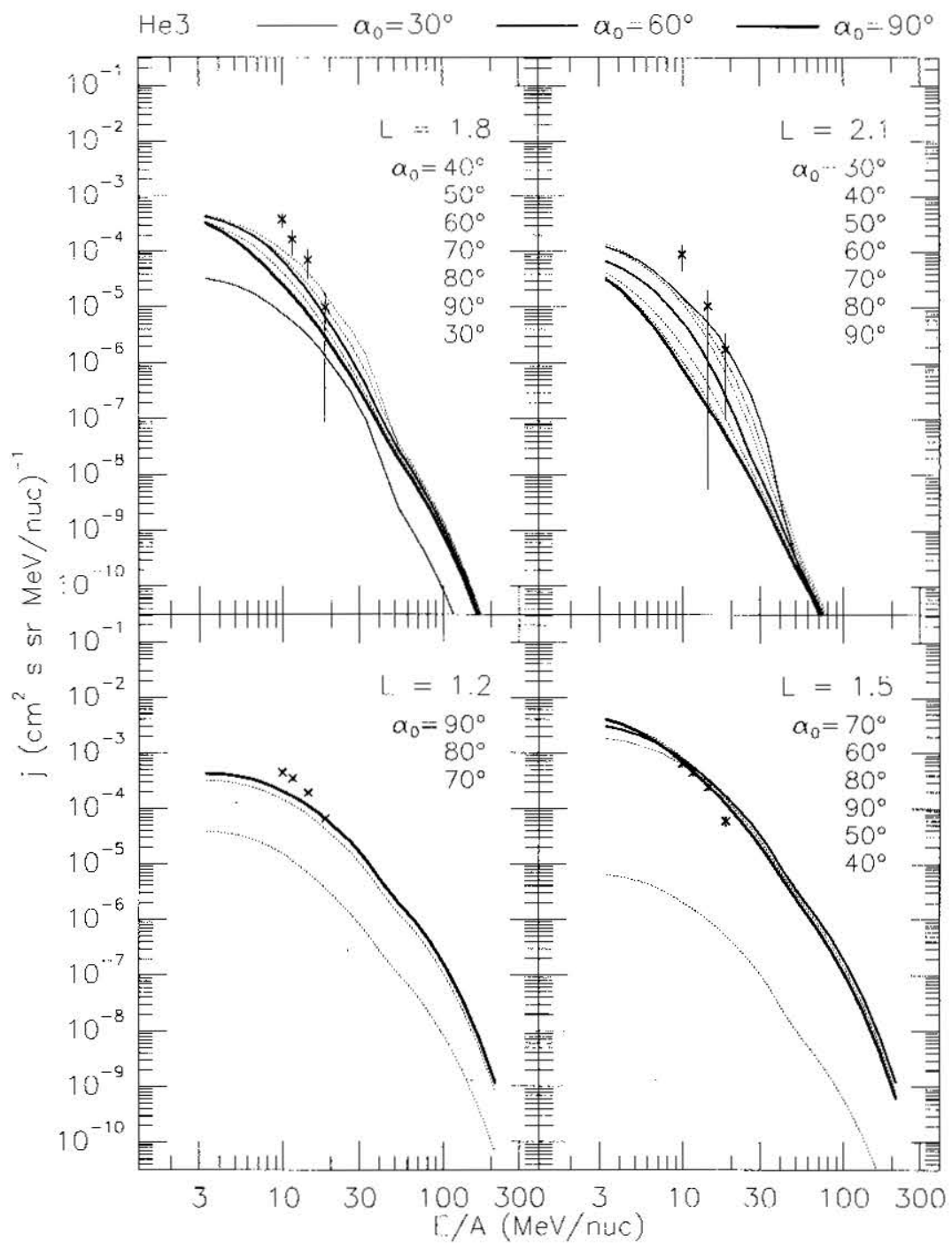

量

Figure 7. Similar to Figure 5 but for ${ }^{3} \mathrm{He}$ from the reaction $\mathrm{p}\left({ }^{4} \mathrm{He}, \mathrm{d}\right){ }^{3} \mathrm{He}$. The data points are from SAMPEX/MAST ${ }^{3} \mathrm{He}$ observations averaged over local pitch angles at $\sim 600 \mathrm{~km}$ altitude [Cummings et al., 1995]. 


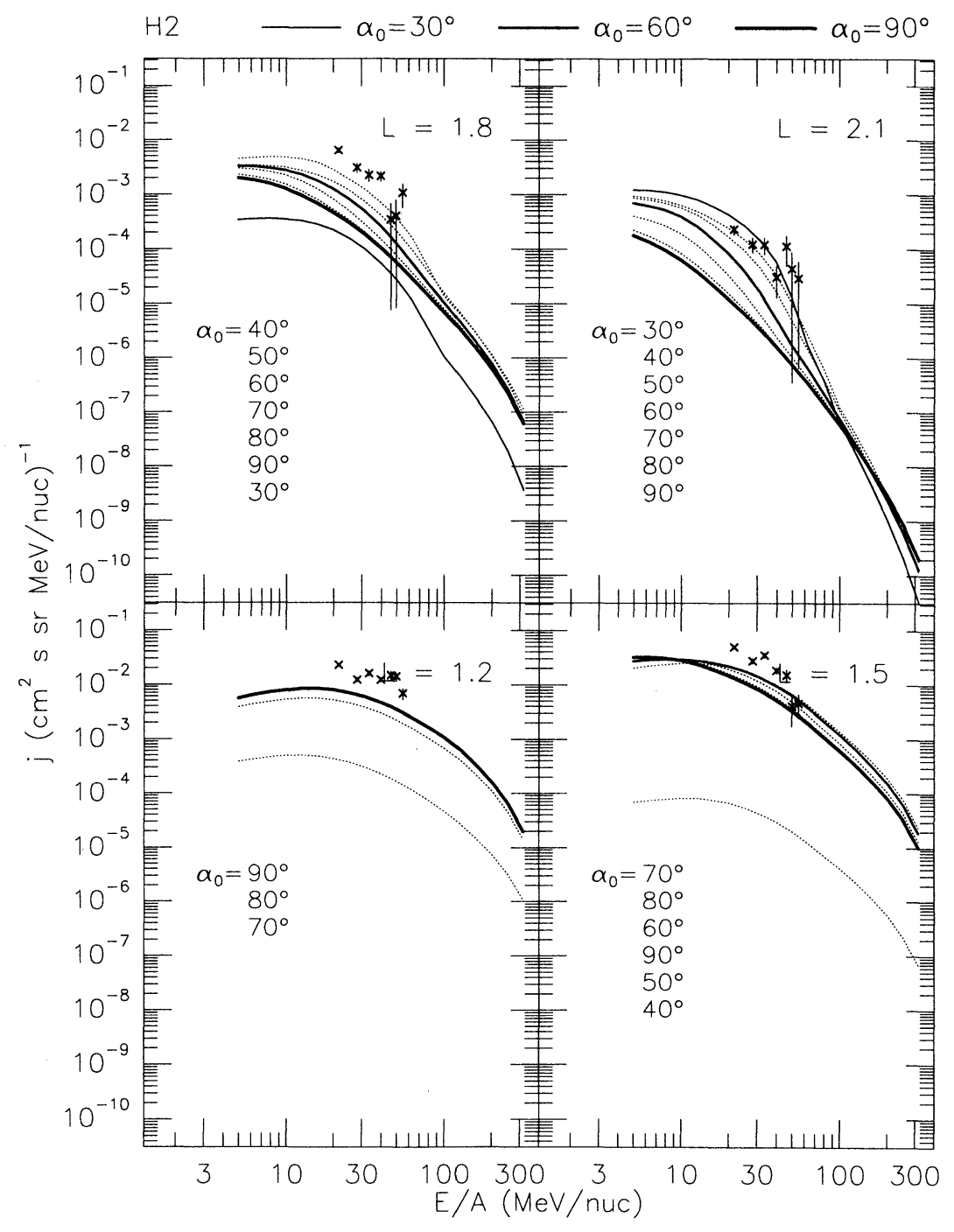

Figure 8. Similar to Figure 5 but for $d$ from the reaction $\mathrm{p}+{ }^{4} \mathrm{He} \rightarrow{ }^{3} \mathrm{He}+\mathrm{d}$. The data points are from SAMPEX/PET d observations at $90^{\circ}$ local pitch angle and $\sim 700 \mathrm{~km}$ altitude [Looper et al., 1995].

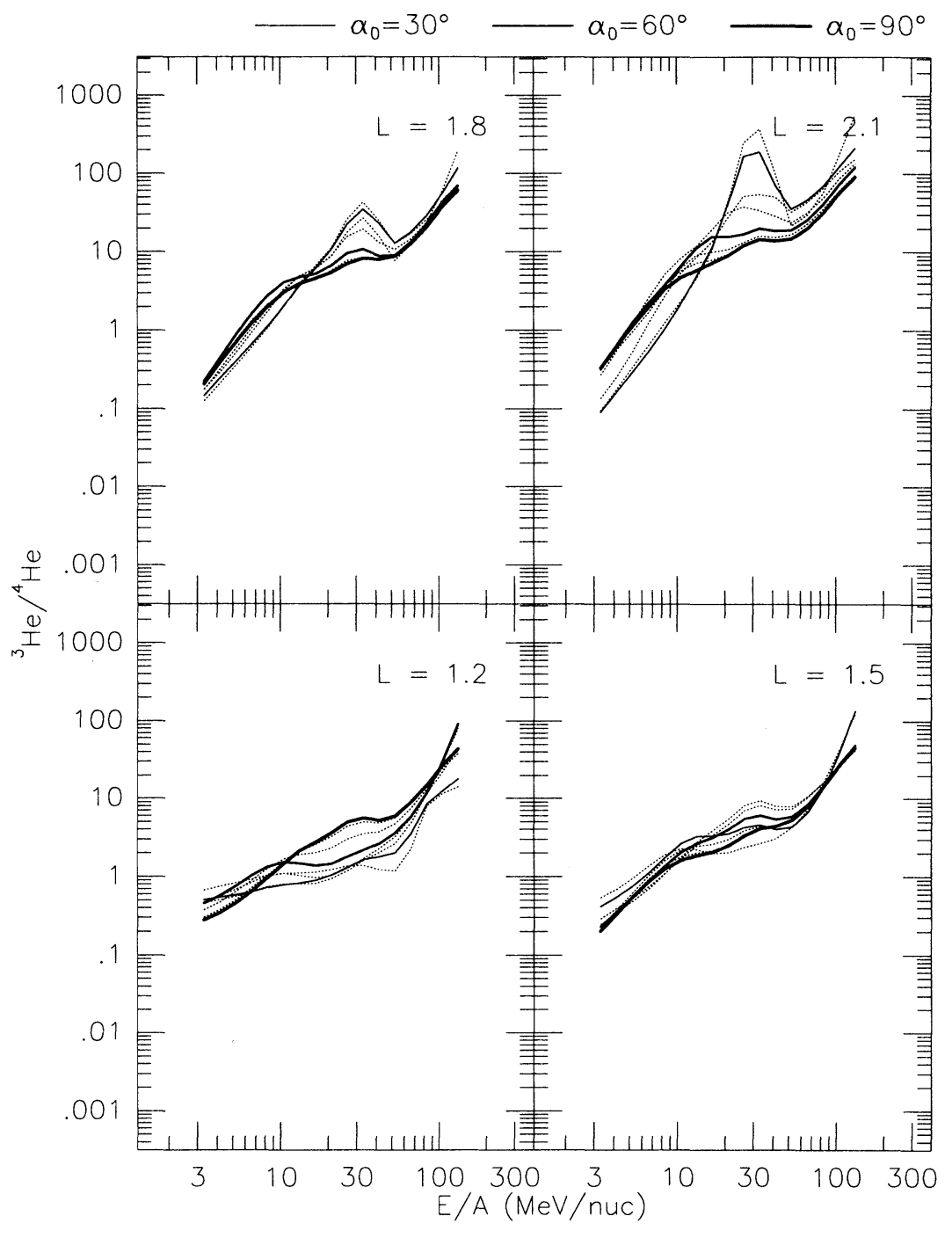

盗

Figure 9. Intensity ratios of ${ }^{3} \mathrm{He}$ and ${ }^{4} \mathrm{He}$ due to the reactions $\mathrm{p}\left({ }^{4} \mathrm{He}, \mathrm{p}\right){ }^{4} \mathrm{He}$ and $\mathrm{p}\left({ }^{4} \mathrm{He}, \mathrm{d}\right){ }^{3} \mathrm{He}$ from Figures 7 and 5 . 


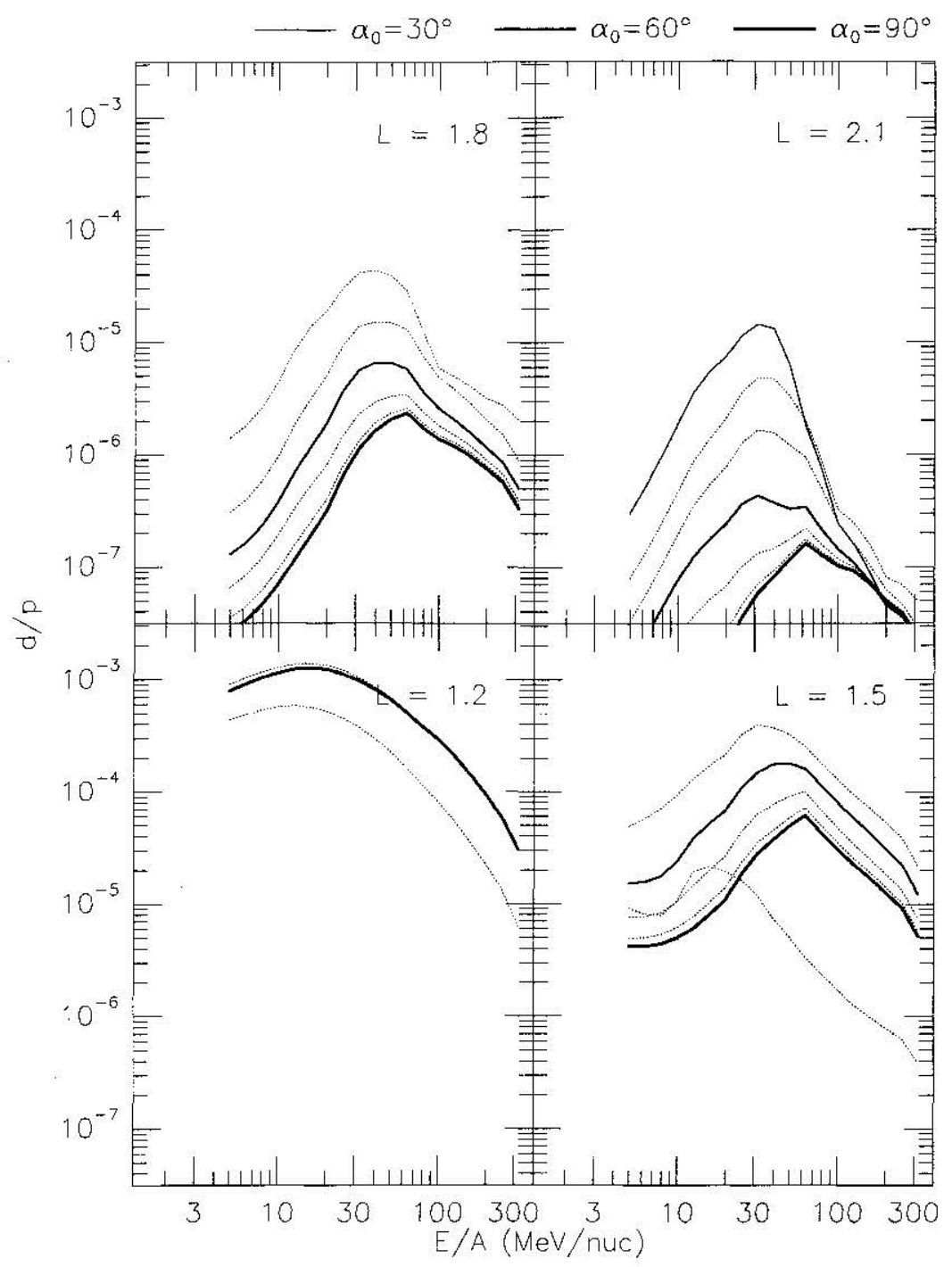

Figure 10. Intensity ratios of $\mathrm{d}$ due to the reaction $\mathrm{p}\left({ }^{4} \mathrm{He}, \mathrm{d}\right)^{3} \mathrm{He}$ and $\mathrm{p}$ from Figures 8 and 3 .

The $e$-folding energy is $E_{0}$ and $H$ is a unit step function. The values of $E_{0}$ are taken from the angle-integrated spectra of Wu et al. and are $15.9 \mathrm{MeV}$ for ${ }^{4} \mathrm{He}, 29.5$ $\mathrm{MeV}$ for ${ }^{3} \mathrm{He}$, and $77.8 \mathrm{MeV}$ for $\mathrm{d}$. The total cross sections $\sigma\left(E_{p}\right)$ are taken from Cucinotta et al. [1996] and are shown in Figure 11.

The source function based on the approximation (11) is

$$
S=\int d E_{p} n j_{p} \sigma F
$$

where $n$, the drift averaged $\mathrm{O}$ density, and $j_{p}$ are evaluated at $\alpha_{p}=\alpha$. The resulting ${ }^{4} \mathrm{He},{ }^{3} \mathrm{He}$, and $\mathrm{d}$ intensities are shown in Figures 12, 13, and 14, respectively. Atmospheric $\mathrm{O}$ appears to be a significant source at $L=1.2$ only. At the higher $L$ shells the $O$ products are significant only in a narrow range of equatorial pitch angles near the edges of the loss cones, corresponding to the altitude range where $\mathrm{O}$ is the dominant component of the atmosphere. However, because of the differing energy spectra (different values of $E_{0}$ ) the atmospheric $O$ source is relatively more significant for $d$ and less significant for ${ }^{4} \mathrm{He}$, compared with ${ }^{3} \mathrm{He}$. Compared with the atmospheric He source at $L=1.2$, atmospheric $\mathrm{O}$ appears to be a comparable source of ${ }^{4} \mathrm{He}$ and $\mathrm{d}$, and a dominant source of ${ }^{3} \mathrm{He}$. However, the $\mathrm{O}$ source is uncertain due to the lack of cross section data and the forward scattering approximation (11) probably leads to an overestimate of the trapped particle source rate.

\section{Discussion}

The calculations described above have no free parameters, depending only on the properties of the proton radiation belt, the upper atmosphere, and the interaction cross sections. If these properties are described accurately and there are no significant additional sources or losses, then the secondary populations must exist at the levels predicted. Although observations of radiation belt light elements with isotopic composition are still quite limited, they do provide some useful comparisons for testing the model and determining whether the atmospheric sources are the predominant ones. 


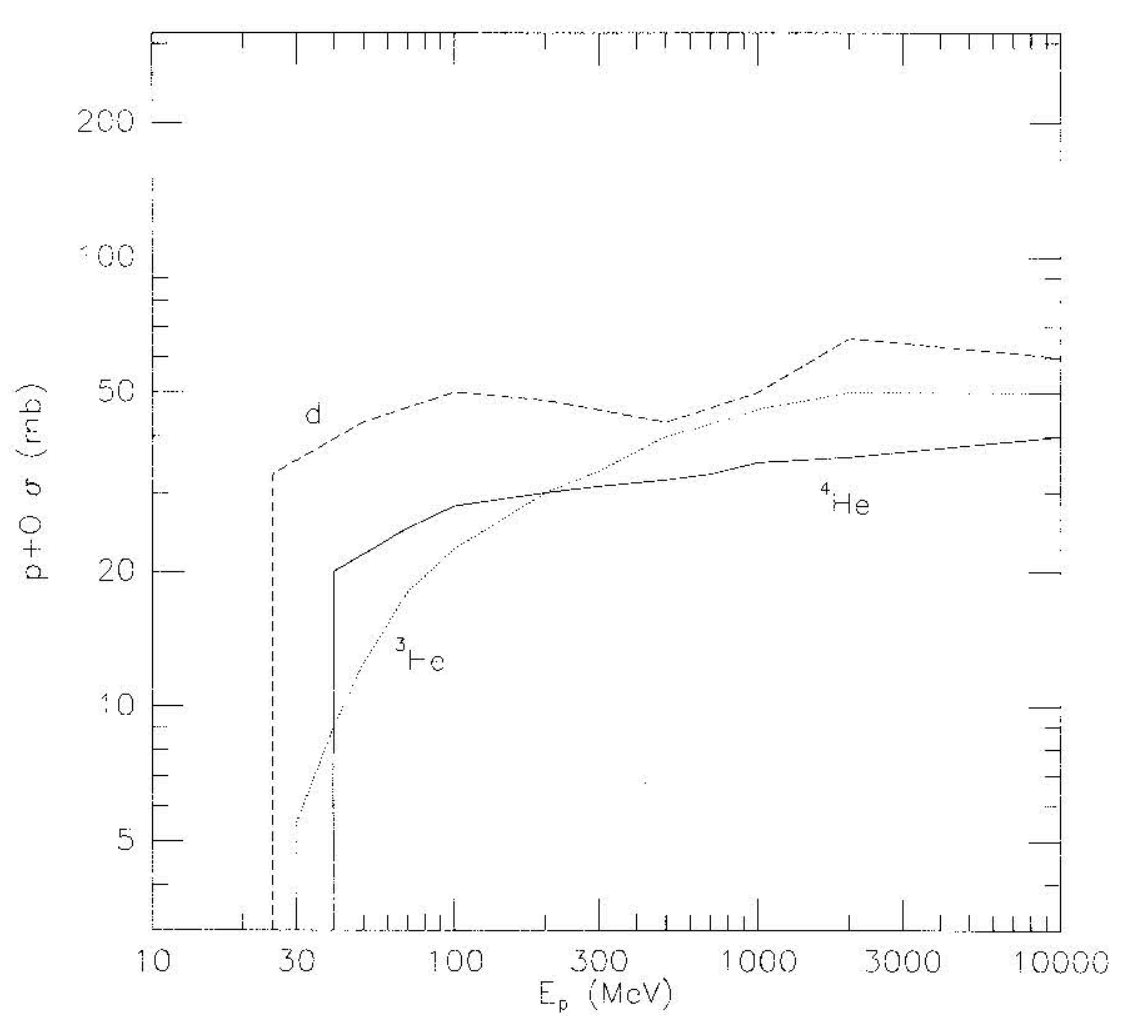

Figure 11. Cross sections for knockout production of ${ }^{3} \mathrm{He},{ }^{4} \mathrm{He}$, and d from CNO (data compiled by Cucinotta et al., [1995]).

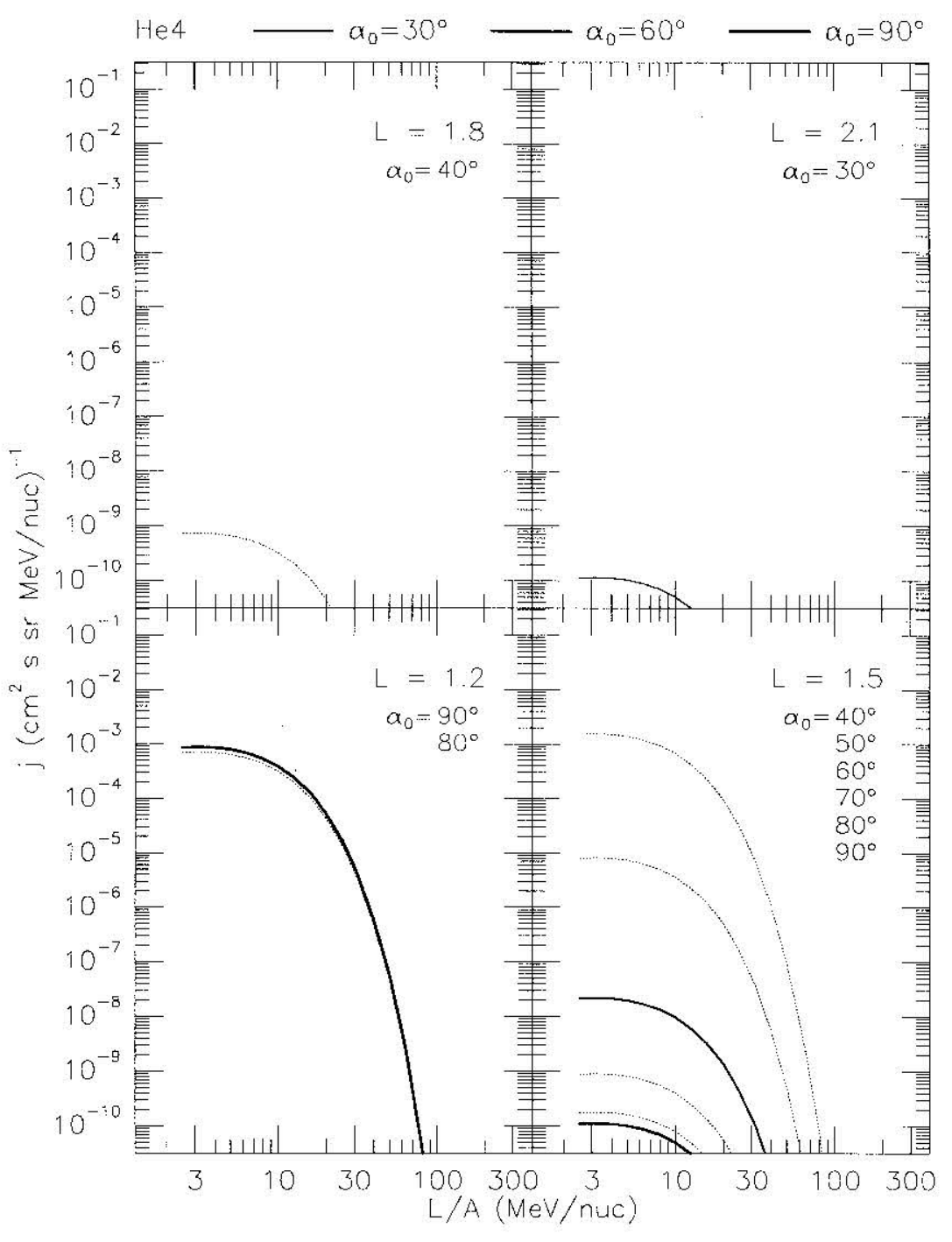

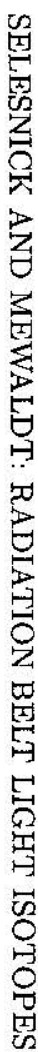

Figure 12. Similar to Figure 5 but for ${ }^{4} \mathrm{He}$ production by $\mathrm{p}$ knockout from $\mathrm{O}$. 


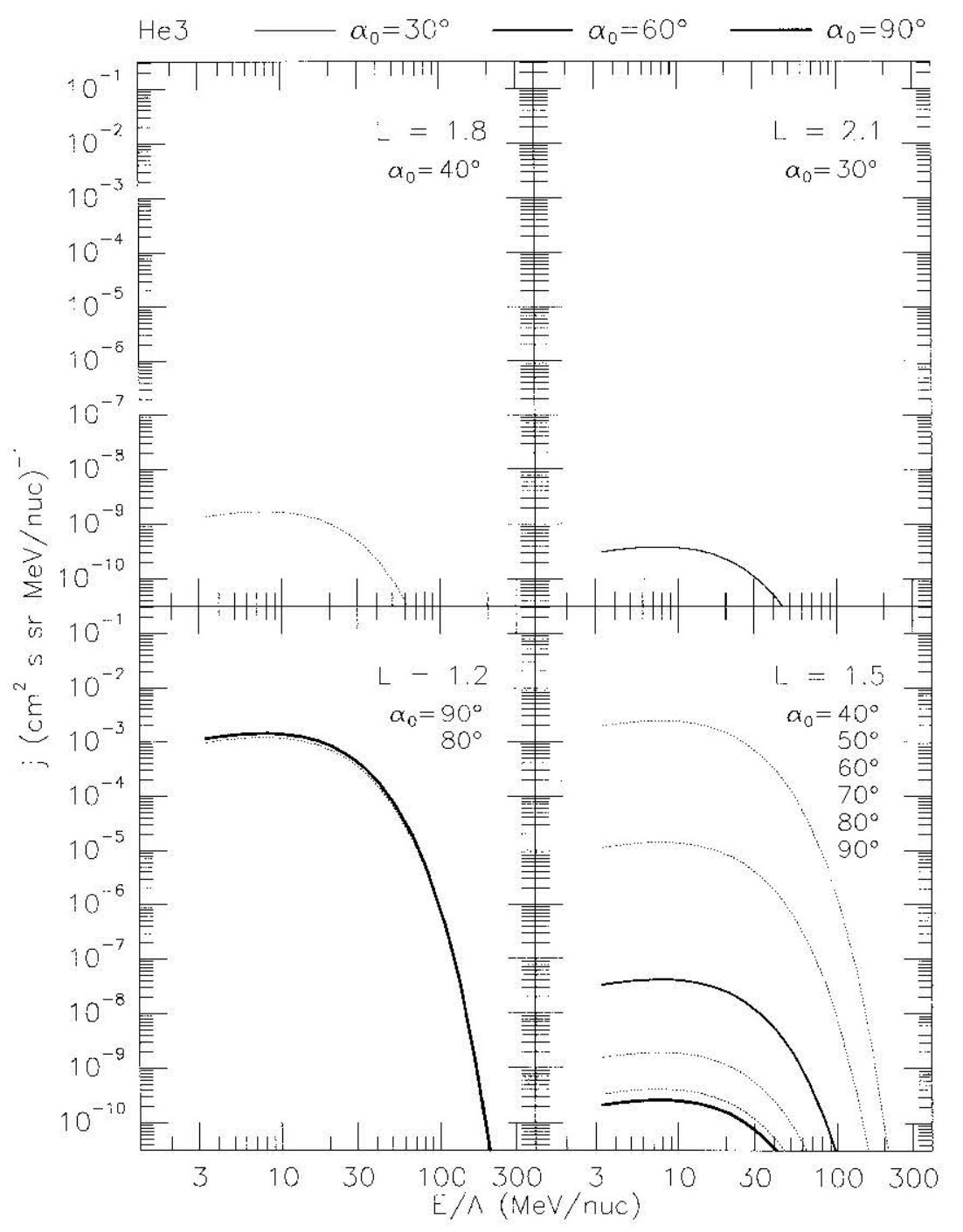

Figure 13. Similar to Figure 12 but for ${ }^{3} \mathrm{He}$ production.

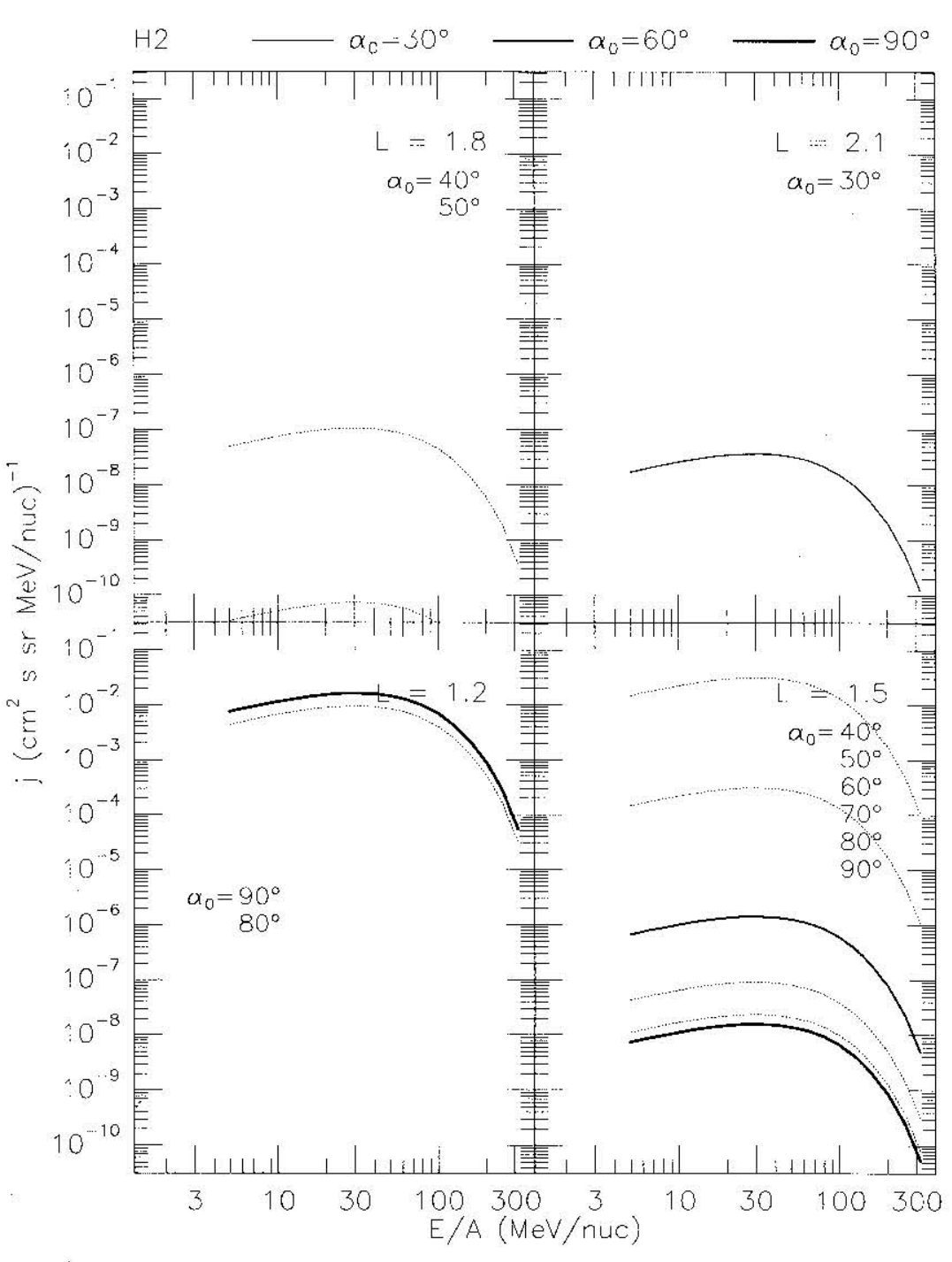

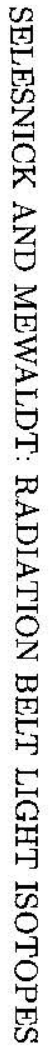

Figure 14. Similar to Figure 12 but for d production. 
As shown in Figures 5, 7, and 8, the atmospheric He source is adequate to account for a substantial fraction of the intensity levels observed on SAMPEX. This is particularly true for ${ }^{3} \mathrm{He}$. For ${ }^{4} \mathrm{He}$ the atmospheric source is adequate at $L=1.2$, but an additional source is required at higher $L$ shells. For d, the atmospheric source appears to bc somewhat lower than the data. The model ${ }^{3} \mathrm{He}$ to ${ }^{4} \mathrm{He}$ ratio at $L=1.2$ is also in reasonable agreement with that observed near $50 \mathrm{MeV} /$ nucleon from the CRRES satellite [Wefel et al,, 1995$]$.

While the elastic scattering and pick-up reactions involving atmospheric He are reasonably well understood, the lack of experimental cross section data for the reactions with atmospheric $O$ lead to considerable uncertainty in the importance of this source. The model results in Figures 12 to 14 should be regarded as upper limits due to the nature of the approximations involved. If the model intensities from the $\mathrm{He}$ and $\mathrm{O}$ sources are combined, then they result in significantly more ${ }^{4} \mathrm{He}$ and especially ${ }^{3} \mathrm{He}$ than are observed in the SAMPEX data at $L=1.2$. This is probably a result of the uncertain cross section model, but it may also be due to additional loss mechanisms that are not included in the calculation. The combined intensities for $\mathrm{d}$ are comparable with the SAMPEX data at $L=1.2$. Despite the uncertainties, it appears that atmospheric $O$ can be a significant source for the inner radiation belt near $L=1.2$. It is not significant at larger $L$ shells because $\mathrm{O}$ is too minor an atmospheric constituent.

It may be possible to distinguish between various atmospheric sources if high resolution pitch angle distributions were observed from a near equatorial satellite. As shown in the results described above, the intensity from an atmospheric source generally peaks at a pitch angle corresponding to the altitude at which this source is the dominant atmospheric constituent. Because SAMPEX orbits at an altitude where $\mathrm{He}$ is dominant in the atmosphere, the maximum intensities from this source were observed near their mirror points.

Reactions that we have not considered may also be significant, such as $p(p, d) \pi$ for producing high energy $d$ or similar reactions on $\mathrm{He}$ and $\mathrm{O}$, while elastic scattering of atmospheric constituents other than $\mathrm{He}$, such as $\mathrm{H}$ and $\mathrm{O}$, may also be significant sources for their corresponding radiation belt components. Tritium could be produced at comparable levels to ${ }^{3} \mathrm{He}$ by reactions on $\mathrm{He}$ and $\mathrm{O}$, and if detected could provide a measurement of radiation belt lifetimes due to its $\sim 12$ year halflife. Fragmentation of atmospheric $\mathrm{O}$ and $\mathrm{N}$ could also produce heavier elements such as $\mathrm{Li}, \mathrm{Be}$, and $\mathrm{B}$. Other possible improvements in the calculation would be to use an updated model for the radiation belt proton intensities based on recent SAMPEX and CRRES data [Looper et al., 1995; Gussenhoven et al., 1993], to include solar cycle variations, to evaluate the time dependence in cases where the lifetimes are comparable to the solar cycle time [Jentsch and Wibberenz, 1980], and to evaluate the role of radial diffusion, which is probably significant at the higher $L$ shells [Jentsch, 1981]. Such refinements will probably be warranted when more detailed observations become available. However, it is already clear that the atmosphere is a significant and possibly dominant source of $\mathrm{He}$ and $\mathrm{H}$ isotopes for the inner radiation belt.

Acknowledgments. We thank J. R. Cummings for the SAMPEX He data, M. D. Looper and J. B. Blake for the SAMPEX $\mathrm{H}$ data, and $\mathrm{K}$. Langanke for help with the cross section data. This work was supported by NASA under contract NAS5-30704 and grant NAGW-1919.

The editor thanks R. B. McKibben and D. G. Mitchell for their assistance in evaluating this paper.

\section{References}

Alons, P. W. F., et al., ${ }^{4} \mathrm{He}(\mathrm{p}, \mathrm{d})^{3} \mathrm{He}$ reaction at 200 and 400 $\mathrm{MeV}$, Phys. Rev., C33, 406-411, 1986.

Comparat, V., R. Frascaria, N. Fujiwara, N. Marty, M. Morlet, P. G. Roos, and A. Willis, Elastic proton scattering on ${ }^{4} \mathrm{He}$ at $156 \mathrm{MeV}$, Phys. Rev. C Nucl. Phys., 12, 251-255, 1975.

Cucinotta, F. A., L. W. Townsend, J. W. Wilson, J. L. Shinn, G. D. Badhwar, and R. R. Dubey, Light ion components of the galactic cosmic rays: nuclear interactions and transport theory, Adv. Space Res., 17(2), 77-86, 1996.

Cummings, J. R, R. A. Mewaldt, R. S. Selesnick, E. C. Stone, J. B. Blake, and M. D. Looper, MAST observations of high enery trapped helium nuclei (abstract), Eos Trans. 76, $A G U$, Fall Meet. Suppl., F501, 1995.

Dragt, A. J, Solar cycle modulation of the radiation belt proton flux, J. Geophys. Res., 76, 2313-2344, 1971.

Farley, T. A., and M. Walt, Source and loss processes of protons in the inner radiation belt, J. Geophys. Res., 76, $8223-8241,1971$

Fong, J., et al., $\mathrm{p}^{4} \mathrm{He}$ Elastic scattering at $788 \mathrm{MeV}$, Phys. Lett., 78B, 205-208, 1978.

Gussenhoven, M. S., E. G. Mullen, M. D. Violet, C. Hein, J. Bass, and D. Madden, CRRES high energy proton flux maps, IEEE Trans. Nucl. Sci., 40, 1450-1457, 1993.

Hedin, A. E., MSIS-86 thermospheric model, J. Geophys. Res., 92, 4649-4662, 1987.

Imai, K., K. Hatanaka, H. Shimizu, N. Tamura, K. Egawa, K. Nisimura, T. Saito, H. Sato, and Y. Wakuta, Polarization and cross section measurements for $\mathrm{p}^{-4} \mathrm{He}$ elastic scattering at 45, 52, 60, and $65 \mathrm{MeV}$, Nucl. Phys. A, 325, 397-407, 1979.

Jentsch, V, On the role of external and internal source in generating energy and pitch angle distributions of innerzone protons, J. Geophys. Res., 86, 701-710, 1981.

Jentsch, V, and G. Wibberenz, An analytic study of the energy and pitch angle distribution of inner-zone protons, J. Geophys. Res., 85, 1-8, 1980.

Langel, R. A., International geomagnetic reference field, 1991 revision, J. Geomagn. Geoelectr., 43, 1007-1012, 1991.

Looper, M. D., J. B. Blake, J. R. Cummings, R. A. Mewaldt, and $R$. S. Selesnick, Maps of hydrogen isotopes at low altitudes in the inner zone of the earth's magnetosphere (abstract), Eos Trans. AGU, 76, Fall Meet. Suppl., F501, 1995 .

McCamis, R. H., J. M. Cameron, L. G. Greeniaus, D. A. Hutcheon, C. A. Miller, M. S. de Jong, B. T. Murdoch, W. T. H. van Oers, J. G. Rogers, and A. W. Stetz, Large 
angle cross sections and analyzing power for proton- ${ }^{4} \mathrm{He}$ elastic scattering between 185 and $500 \mathrm{MeV}$, Nucl. Phys. A, 302, 388-400, 1978.

Meyer, J. P., Deuterons and $\mathrm{He}^{3}$ formation and destruction in proton induced spallation of light nuclei $(Z<8)$, Astron. Astrophys. Suppl., 7, 417-467, 1972.

Moss, G. A., et al., Proton- ${ }^{4} \mathrm{He}$ elastic scattering at intermediate energies, Phys. Rev. C Nucl. Phys., 21, 1932-1943, 1980.

Salamon, M. H., A range-energy program for relativistic heavy ions in the region $1<E<3000 \mathrm{MeV} / \mathrm{amu}$, report, Lawrence Berkeley Lab., Univ. of Calif., 1980.

Votta, L. G., P. G. Roos, N. S. Chant, and R. Woody, III, Elastic protons scattering from ${ }^{3} \mathrm{He}$ and ${ }^{4} \mathrm{He}$ and the ${ }^{4} \mathrm{He}(\mathrm{p}, \mathrm{d})^{3} \mathrm{He}$ reaction at $85 \mathrm{MeV}$, Phys. Rev. C Nucl. Phys., 10, 520-528, 1974.

Wefel, J. P., J. Chen, J. F. Cooper, T. G. Guzik, and
K. R. Pyle, The isotopic composition of geomagnetically trapped helium, Proc. Int. Cosmic Ray Conf. 24th, 4, 1021-1024, 1995.

Wu, J. R., C. C. Chang, and H. D. Holmgren, Chargedparticle spectra: $90 \mathrm{MeV}$ protons on ${ }^{27} \mathrm{Al},{ }^{58} \mathrm{Ni},{ }^{90} \mathrm{Zr}$, and ${ }^{209} \mathrm{Bi}$, Phys. Rev. C Nucl. Phys., 19, 698-713, 1979.

R. A. Mewaldt, California Institute of Technology, mail code 220-47, Pasadena, CA 91125. (e-mail: dick@srl.caltech.edu).

R. S. Selesnick, The Aerospace Corporation, M2259, P.O. Box 92957, Los Angeles, CA 90009. (e-mail: rich_selesnick@qmail2.aero.org).

(Received March 12, 1996; revised May 16, 1996; accepted May 23, 1996.) 